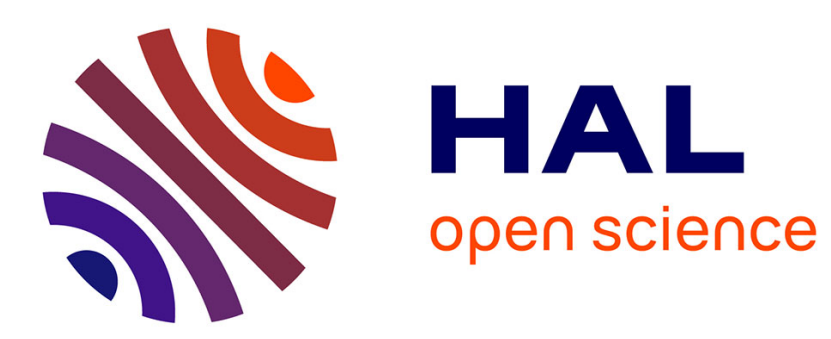

\title{
Parameter and time-delay identification of continuous-time models from non-uniformly sampled data
}

Fengwei Chen, Hugues Garnier, Marion Gilson

\section{To cite this version:}

Fengwei Chen, Hugues Garnier, Marion Gilson. Parameter and time-delay identification of continuoustime models from non-uniformly sampled data. 53rd IEEE Conference on Decision and Control, CDC 2014, Dec 2014, Los Angeles, United States. hal-01066495

\section{HAL Id: hal-01066495 \\ https://hal.science/hal-01066495}

Submitted on 21 Sep 2014

HAL is a multi-disciplinary open access archive for the deposit and dissemination of scientific research documents, whether they are published or not. The documents may come from teaching and research institutions in France or abroad, or from public or private research centers.
L'archive ouverte pluridisciplinaire HAL, est destinée au dépôt et à la diffusion de documents scientifiques de niveau recherche, publiés ou non, émanant des établissements d'enseignement et de recherche français ou étrangers, des laboratoires publics ou privés. 


\title{
Parameter and Time-delay Identification of Continuous-time Models from Non-uniformly Sampled Data
}

\author{
Fengwei Chen, Hugues Garnier, Marion Gilson
}

\begin{abstract}
This paper considers the problem of continuous-time model identification with arbitrary time-delay from irregularly sampled data. The proposed method estimates the plant and the time-delay in a separable way, when estimating one of them, the other is assumed to be fixed. More precisely, the plant is estimated by the iterative instrumental variable SRIVC method while the time-delay is estimated by the Gauss-Newton method. Because of the nonlinear relationship between the loss function and the time-delay, a low-pass filter is employed to extend the global convergence region for the time-delay estimation. Numerical examples are presented to illustrate the properties of the proposed method.
\end{abstract}

\section{INTRODUCTION}

Irregularly sampled data occurs quite frequently in many application areas. For example, in astronomy, particularly in stellar physics, the obtained data always has unknown periodicity. In even-based sampling (e.g. Lebesgue sampler), the sampler is triggered by the arrival of a certain event process. When one wants to model a system from irregularly sampled data, the use a continuous-time (CT) model is preferable, since the CT model parameters are independent of the sampling period (see e.g. [5], [2]).

Many processes have intrinsic delay, as it is the case in thermal and chemical processes, therefore it is a common practice to assume a time-delay in the model. The problem of time-delay estimation based on a discrete-time (DT) model has been studied a lot. Usually the time-delay is assumed to be an integer number of the sampling period, thus the delayed output of the system can be obtained by adjusting the phase-shift. However, this flexibility is lost in the situation of non-uniform sampling because of the time-varying sampling intervals. To handle irregularly sampled data, the algorithm must be capable of estimating arbitrary time-delay.

Several methods have been proposed for CT model parameter and time-delay estimation. A linear filter-based method was introduced in [1], where the time-delay along with the transfer function parameters are estimated in an iterative way through simple linear regression. Iterative global nonlinear least-squares and instrumental variable methods were suggested in [10], where the plant parameters and time-delay are estimated in a separable way. More recently, a wavelet correlation method was introduced in [8] to estimate the time-delay for MIMO dynamical systems, by calculating and

Fengwei Chen, Hugues Garnier and Marion Gilson are with the Centre de Recherche en Automatique de Nancy, Université de Lorraine, CNRS, 2 rue Jean Lamour, 54519 Vandœuvre-lèsNancy, France. Email: fengwei.chen, hugues.garnier, marion.gilson@univ-lorraine.fr handling the cross-correlation between the CWT coefficients of the input and the output data.

In this paper, the time-delay and the plant parameters are also estimated in a separable way, but a low-pass filter is introduced to improve the convergence performance for the time-delay estimation (see [3]). More precisely, the plant and the time-delay are estimated by the iterative instrumental variable (IV) and the Gauss-Newton methods, respectively. For the IV method, the simplified refined instrumental variable method for CT systems (SRIVC) is adopted. It was first developed by Young and Jakeman in the open-loop situation [12] and recent developments can be found in [4], [11].

This paper is organized in the following way. We first define the parameter estimation problem in Section II. Subsequently, the SRIVC method for plant parameter identification is recalled in Section III. Then the time-delay estimation problem is discussed in Section IV. The final algorithm for time-delay system identification is detailed in Section V. Finally, in Section VI, numerical examples are presented to illustrate the properties of the proposed method.

\section{Problem Statement}

Consider the following CT output error (COE) model

$$
\left\{\begin{array}{l}
x(t)=G\left(p, \boldsymbol{\theta}^{o}\right) u\left(t-\tau^{o}\right)=\frac{B\left(p, \boldsymbol{\theta}^{o}\right)}{A\left(p, \boldsymbol{\theta}^{o}\right)} u\left(t-\tau^{o}\right) \\
y\left(t_{k}\right)=x\left(t_{k}\right)+e\left(t_{k}\right)
\end{array}\right.
$$

where $u(t), x(t)$ and $\tau^{o}$ are the excitation signal, the noisefree response and the time-delay, respectively. $p$ denotes the differentiation operator, i.e. $p x=d x / d t . B\left(p, \boldsymbol{\theta}^{o}\right)$ and $A\left(p, \boldsymbol{\theta}^{\circ}\right)$ are polynomials assumed to be coprime of the following form

$$
\begin{aligned}
& B\left(p, \boldsymbol{\theta}^{o}\right)=b_{0}^{o} p^{m}+b_{1}^{o} p^{m-1}+\cdots+b_{m}^{o} \\
& A\left(p, \boldsymbol{\theta}^{o}\right)=a_{0}^{o} p^{n}+a_{1}^{o} p^{n-1}+\cdots+1 \quad(n>m)
\end{aligned}
$$

The unknown parameters are stacked columnwise in

$$
\boldsymbol{\theta}^{o}=\left[\begin{array}{llllll}
a_{0}^{o} & \cdots & a_{n-1}^{o} & b_{0}^{o} & \cdots & b_{m}^{o}
\end{array}\right]^{T}
$$

The input-output signals of the system are observed at irregular time-instant $t_{k}$, for $k=1,2, \cdots, N$, the sampling interval is denoted as

$$
h_{k}=t_{k+1}-t_{k}
$$

In most practical measurement situations, it is reasonable to consider that $x\left(t_{k}\right)$ is corrupted by a DT measurement noise $e\left(t_{k}\right)$, which is assumed to be white here. The identification problem can be stated as: assume the orders $n$ and $m$ are 
known, the identification objective is to estimate the unknown parameters and time-delay from irregularly sampled data $Z^{N}=\left\{u\left(t_{k}\right) ; y\left(t_{k}\right)\right\}_{k=1}^{N}$.

\section{Instrumental VARiable Method FOR Plant PARAMETER ESTIMATION}

Amongst the different implementation of the optimal IV approach, the SRIVC method is known to be one of the most effective and is used here to identify the CT transfer function parameters. In this section, the time-delay is assumed to be known $\left(\tau=\tau^{o}\right)$. The SRIVC method makes use of a low-pass filter to generate the (filtered) input/output timederivatives of the differential equation model. The filter $F(p)$ takes the following form

$$
F\left(p, \hat{\boldsymbol{\theta}}^{j}\right)=\frac{1}{A\left(p, \hat{\boldsymbol{\theta}}^{j}\right)}
$$

Then, the COE model can be equivalently reformulated in the following regression form

$$
y_{F}\left(t_{k}, \hat{\boldsymbol{\theta}}^{j}\right)=\boldsymbol{\phi}_{F}^{T}\left(t_{k}, \hat{\boldsymbol{\theta}}^{j}, \tau^{o}\right) \hat{\boldsymbol{\theta}}^{j}+\varepsilon\left(t_{k}, \hat{\boldsymbol{\theta}}^{j}, \tau^{o}\right)
$$

with

$$
\begin{array}{r}
\boldsymbol{\phi}_{F}^{T}\left(t_{k}, \hat{\boldsymbol{\theta}}^{j}, \tau^{o}\right)=\left[-y_{F}^{(n)}\left(t_{k}, \hat{\boldsymbol{\theta}}^{j}\right) \cdots-y_{F}^{(1)}\left(t_{k}, \hat{\boldsymbol{\theta}}^{j}\right),\right. \\
\left.u_{F}^{(m)}\left(t_{k}-\tau^{o}, \hat{\boldsymbol{\theta}}^{j}\right) \cdots u_{F}\left(t_{k}-\tau^{o}, \hat{\boldsymbol{\theta}}^{j}\right)\right]
\end{array}
$$

where $\varepsilon\left(t_{k}, \hat{\boldsymbol{\theta}}^{j}, \tau^{o}\right)$ is the equation error, $(\cdot)_{F}$ denotes the filtering operation, i.e. $(\cdot)_{F}=F\left(p, \hat{\boldsymbol{\theta}}^{j}\right)(\cdot)$. The IV vector $\boldsymbol{\psi}_{F}^{T}\left(t_{k}, \hat{\boldsymbol{\theta}}^{j}, \tau^{o}\right)$ is chosen as

$$
\begin{array}{r}
\boldsymbol{\psi}_{F}^{T}\left(t_{k}, \hat{\boldsymbol{\theta}}^{j}, \tau^{o}\right)=\left[-x_{F}^{(n)}\left(t_{k}, \hat{\boldsymbol{\theta}}^{j}, \tau^{o}\right) \cdots-x_{F}^{(1)}\left(t_{k}, \hat{\boldsymbol{\theta}}^{j}, \tau^{o}\right)\right. \\
\left.u_{F}^{(m)}\left(t_{k}-\tau^{o}, \hat{\boldsymbol{\theta}}^{j}\right) \cdots u_{F}\left(t_{k}-\tau^{o}, \hat{\boldsymbol{\theta}}^{j}\right)\right]
\end{array}
$$

where $x\left(t_{k}, \hat{\boldsymbol{\theta}}^{j}, \tau^{o}\right)$ is the noise-free response of the following auxiliary model

$$
x\left(t_{k}, \hat{\boldsymbol{\theta}}^{j}, \tau^{o}\right)=\frac{B\left(p, \hat{\boldsymbol{\theta}}^{j}\right)}{A\left(p, \hat{\boldsymbol{\theta}}^{j}\right)} u\left(t_{k}-\tau^{o}\right)
$$

From $N$ measured input-output data, the SRIVC parameter estimates are given by

$$
\hat{\boldsymbol{\theta}}^{j+1}=\left[\boldsymbol{\Psi}_{F}\left(\hat{\boldsymbol{\theta}}^{j}, \tau^{o}\right) \boldsymbol{\Phi}_{F}^{T}\left(\hat{\boldsymbol{\theta}}^{j}, \tau^{o}\right)\right]^{-1} \boldsymbol{\Psi}_{F}\left(\hat{\boldsymbol{\theta}}^{j}, \tau^{o}\right) \boldsymbol{y}_{F}\left(\hat{\boldsymbol{\theta}}^{j}\right)
$$

with

$$
\begin{aligned}
& \boldsymbol{y}_{F}\left(\hat{\boldsymbol{\theta}}^{j}\right)=\left[\begin{array}{lll}
y_{F}\left(t_{s}, \hat{\boldsymbol{\theta}}^{j}\right) & \cdots & y_{F}\left(t_{N}, \hat{\boldsymbol{\theta}}^{j}\right)
\end{array}\right]^{T} \\
& \boldsymbol{\Phi}_{F}\left(\hat{\boldsymbol{\theta}}^{j}, \tau^{o}\right)=\left[\begin{array}{llll}
\boldsymbol{\phi}_{F}\left(t_{s}, \hat{\boldsymbol{\theta}}^{j}, \tau^{o}\right) & \cdots & \boldsymbol{\phi}\left(t_{N}, \hat{\boldsymbol{\theta}}^{j}, \tau^{o}\right)
\end{array}\right] \\
& \boldsymbol{\Psi}_{F}\left(\hat{\boldsymbol{\theta}}^{j}, \tau^{o}\right)=\left[\begin{array}{llll}
\boldsymbol{\psi}_{F}\left(t_{s}, \hat{\boldsymbol{\theta}}^{j}, \tau^{o}\right) & \cdots & \boldsymbol{\psi}_{F}\left(t_{N}, \hat{\boldsymbol{\theta}}^{j}, \tau^{o}\right)
\end{array}\right]
\end{aligned}
$$

where $t_{s} \geq \tau^{o}$.

As it can be noted from (9), the SRIVC method uses an adaptive procedure where the parameter estimates are iteratively refined. At each iteration, an auxiliary model is used to generate the instrumental variables and filter based on the parameters obtained at the previous iteration. When performing the CT filtering operation, the intersample behavior is required to reconstruct a 'CT input signal' from the irregularly sampled data. In many situations, as it is the case in computer controlled systems, the intersample behavior of the input $u\left(t_{k}\right)$ is always known, such as piece-wise constant (zero-order hold) or piece-wise linear (first-orderhold). However, the intersample behavior of the sampled output $y\left(t_{k}\right)$ is missing. It is then a common practice to assume $y(t)$ is first-order hold. The 4th-order Runge-Kutta (RK4) method can then be used to compute the filtered timederivatives for the output signal at the required irregular timeinstants.

\section{ExpANSION OF GLOBAL CONVERGENCE REGION IN TIME-DELAY ESTIMATION}

In this section, the plant-independent time-delay estimation is investigated, so $\boldsymbol{\theta}=\boldsymbol{\theta}^{\circ}$ is assumed in the sequel. $\tau$ is assumed to be a 'pure delay' and appears as an explicit parameter in the CT model. With these assumptions, the estimated error can be formulated as

$$
\epsilon\left(t, \boldsymbol{\theta}^{o}, \tau\right)=y(t)-G\left(p, \boldsymbol{\theta}^{o}\right) u(t-\tau)
$$

The time-delay can be estimated by minimizing a certain criterion of $\epsilon\left(t, \boldsymbol{\theta}^{o}, \tau\right)$. Here the energy of the estimated error is considered. The estimate of $\tau$ can be obtained by the following fitting

$$
\hat{\tau}=\arg \min _{\tau} J\left(\boldsymbol{\theta}^{o}, \tau\right)=\arg \min _{\tau} \frac{1}{2} \int_{-\infty}^{\infty} \epsilon^{2}\left(t, \boldsymbol{\theta}^{o}, \tau\right) d t
$$

Numerical methods are typical ways to solve (11), such as the Gauss-Newton or the Levenberg-Marquardt algorithm. Since the cost function $J\left(\boldsymbol{\theta}^{o}, \tau\right)$ is multi-modal with respect to $\tau$, the numerical method suffers from local minima and the final estimate $\hat{\tau}$ is highly dependent on the initial value $\tau^{0}$. To increase the chance of converging to the global minimum, the use of a low-pass filter was suggested in [3]. This result is used in this paper to improve the convergence performance of the proposed method.

Let $L(p)$ be a CT low-pass filter with the cutoff frequency $\omega_{c}^{\mathrm{LF}}$. The estimate $\hat{\tau}$ can be obtained by minimizing the energy of the filtered estimated error

$$
\begin{aligned}
& \hat{\tau}=\arg \min _{\tau} \bar{J}\left(\boldsymbol{\theta}^{o}, \tau\right) \\
& \bar{J}\left(\boldsymbol{\theta}^{o}, \tau\right)=\frac{1}{2} \int_{-\infty}^{\infty}\left[L(p) \epsilon\left(t, \boldsymbol{\theta}^{o}, \tau\right)\right]^{2} d t
\end{aligned}
$$

From the Parseval theorem, $\bar{J}\left(\boldsymbol{\theta}^{o}, \tau\right)$ also has the following frequency-domain interpretation (see e.g. [6], [3])

$$
\begin{aligned}
\bar{J}\left(\boldsymbol{\theta}^{o}, \tau\right)= & \frac{1}{4 \pi} \int_{-\infty}^{+\infty}\left[\left|G\left(i \omega, \boldsymbol{\theta}^{o}\right) e^{-i \omega \tau^{o}}-G\left(i \omega, \boldsymbol{\theta}^{o}\right) e^{-i \omega \tau}\right|^{2}\right. \\
& \left.\times \Phi_{u}(\omega)+\Phi_{e}(\omega)\right]|L(i \omega)|^{2} d \omega
\end{aligned}
$$

where $\Phi_{u}(\omega)$ and $\Phi_{e}(\omega)$ are the power spectral densities (PSD) of $u(t)$ and $e(t)$, respectively.

Let the time-delay error denoted as

$$
\delta \tau=\tau-\tau^{o}
$$


Let us now define $\bar{V}\left(\boldsymbol{\theta}^{\circ}, \tau\right)$ as the noise-free version of (14)

$$
\begin{aligned}
\bar{V}\left(\boldsymbol{\theta}^{o}, \delta \tau\right)= & \frac{1}{4 \pi} \int_{-\infty}^{+\infty}\left|G\left(i \omega, \boldsymbol{\theta}^{o}\right) e^{-i \omega \tau^{o}}\right|^{2}\left|1-e^{-i \omega \delta \tau}\right|^{2} \\
& \times \Phi_{u}(\omega)|L(i \omega)|^{2} d \omega
\end{aligned}
$$

From (16), it can be observed that $\bar{V}\left(\boldsymbol{\theta}^{o}, \delta \tau\right)$ depends on $\delta \tau$ and the following transfer function

$$
T(i \omega)=L(i \omega) G\left(i \omega, \boldsymbol{\theta}^{o}\right) e^{-i \omega \tau^{o}}
$$

Since $T(i \omega)$ requires the knowledge of the true model $G\left(i \omega, \boldsymbol{\theta}^{\circ}\right)$, which is not known in practical situations, the following theorem provides an alternative to compute $\bar{V}\left(\boldsymbol{\theta}^{\circ}, \delta \tau\right)$ from filtered data.

Theorem 1 (see [3]): Assume the PSD of $u(t)$ is denoted by $\Phi_{u}(\omega)$ and let $z(t)$ be the noise-free output of the following filter

$$
T(i \omega)=L(i \omega) G\left(i \omega, \boldsymbol{\theta}^{o}\right) e^{-i \omega \tau^{o}}
$$

Then an estimate of $\bar{V}\left(\boldsymbol{\theta}^{\circ}, \delta \tau\right)$ can be obtained by

$$
\bar{V}\left(\boldsymbol{\theta}^{o}, \delta \tau\right)=R_{z}(0)-R_{z}(\delta \tau)
$$

where $R_{z}$ is the autocorrelation function of $z(t)$.

Proof: $\bar{V}\left(\boldsymbol{\theta}^{\circ}, \delta \tau\right)$ can be given as

$$
\begin{gathered}
\bar{V}\left(\boldsymbol{\theta}^{o}, \delta \tau\right)=\frac{1}{4 \pi} \int_{-\infty}^{+\infty}|T(i \omega)|^{2}\left|1-e^{-i \omega \delta \tau}\right|^{2} \Phi_{u}(\omega) d \omega \\
=\frac{1}{4 \pi} \int_{-\infty}^{+\infty} \Phi_{z}(\omega)\left(2-e^{-i \omega \delta \tau}-e^{i \omega \delta \tau}\right) d \omega
\end{gathered}
$$

where $\Phi_{z}(\omega)=|T(i \omega)|^{2} \Phi_{u}(\omega)$ is the PSD of $z(t)$. By using the well-known Wiener-Khinchin theorem, (19) can be reformulated as

$$
\begin{gathered}
\bar{V}\left(\boldsymbol{\theta}^{o}, \delta \tau\right)=R_{z}(0)-\frac{1}{2} R_{z}(-\delta \tau)-\frac{1}{2} R_{z}(\delta \tau) \\
=R_{z}(0)-R_{z}(\delta \tau)
\end{gathered}
$$

The second equation in (20) uses the fact that $R_{z}(\delta \tau)$ is an even function.

To illustrate the low-pass filtering effects on the global convergence, let us consider the following example.

Example 1: Consider the following system

$$
x(t)=\frac{b_{0}^{o}}{a_{0}^{o} p+1} u\left(t-\tau^{o}\right)
$$

a) The system (with $a_{0}^{o}=0.5, b_{0}^{o}=0.5$ and $\tau^{o}=5$ ) is observed for $N=20,000$ time-instants, the sampling period $h_{k}$ is uniformly distributed in $[0.01,0.09] \mathrm{s}$.

b) To approximate $\Phi_{u}(\omega)=1, u\left(t_{k}\right)$ is chosen to be a zero-mean random sequence with the variance of

$$
\mathbb{E}\left\{u^{2}\left(t_{k}\right)\right\}=1 / h_{k}
$$

c) Three different low-pass filters are considered
1) Filter 1: $L_{1}(i \omega)=1 \quad$ (No filtering)

2) Filter 2: $L_{2}(i \omega)= \begin{cases}1 & |\omega| \leq \omega_{c}^{\mathrm{LF}} \\ 0 & |\omega|>\omega_{c}^{\mathrm{LF}}\end{cases}$

3) Filter 3:

$$
L_{3}(i \omega)= \begin{cases}G^{-1}\left(i \omega, \boldsymbol{\theta}^{o}\right) e^{i \omega \tau^{o}} & |\omega| \leq \omega_{c}^{\mathrm{LF}} \\ 0 & |\omega|>\omega_{c}^{\mathrm{LF}}\end{cases}
$$

Actually the cut-off frequency $\omega_{c}^{\mathrm{LF}}$ of $L(i \omega)$ can take any positive value, given the fact the purpose of the low-pass filtering is to extend the global convergence region. It is then advised to choose $\omega_{c}^{\mathrm{LF}}$ to be lower than the system bandwidth $\omega_{b}$. In this example $\omega_{c}^{\mathrm{LF}}=$ $0.25 \mathrm{rad} / \mathrm{s}$ while $\omega_{b}=0.5 \mathrm{rad} / \mathrm{s}$.

Note that when filter $L_{3}(i \omega)$ is chosen, $T(i \omega)$ becomes an ideal low-pass filter. $\bar{V}\left(\boldsymbol{\theta}^{o}, \delta \tau\right)$ has then the following timedomain representation (see [3])

$$
\begin{aligned}
\bar{V}\left(\boldsymbol{\theta}^{o}, \delta \tau\right) & =\frac{1}{4 \pi} \int_{-\omega_{c}^{\mathrm{LF}}}^{\omega_{c}^{\mathrm{LF}}}\left|1-e^{-i \omega \delta \tau}\right|^{2} d \omega \\
& =\frac{\omega_{c}^{\mathrm{LF}}}{\pi}-\frac{\sin \left(\omega_{c}^{\mathrm{LF}} \delta \tau\right)}{\pi \delta \tau}
\end{aligned}
$$

Let $\delta \tau_{\max }$ denote the maximum delay uncertainty (or admissible region uncertainty), for which $|\delta \tau| \leq \delta \tau_{\max }$, such that $\bar{V}\left(\boldsymbol{\theta}^{\circ}, \delta \tau\right)$ is unimodal. Equation (22) can then be transformed to make a cardinal sine function appear

$$
r=\sin (t) / t
$$

A cardinal sine function has many extrema. An approximation of the $t$-coordinate $(t \geq 0)$ of the $i$-th extremum is

$$
t_{i}=(i+1 / 2) \pi-\frac{1}{(i+1 / 2) \pi}
$$

Because $t_{0}=0$ is the global minimum, $t_{1}$ can then be used to compute the maximum delay uncertainty $\delta \tau_{\max }$. By equating $t_{1}$ to $\omega_{c}^{\mathrm{LF}} \delta \tau_{\max }^{\mathrm{L} 3}$, we have

$$
\delta \tau_{\max }^{\mathrm{L} 3} \approx t_{1} / \omega_{c}^{\mathrm{LF}} \approx 4.5 / \omega_{c}^{\mathrm{LF}}
$$

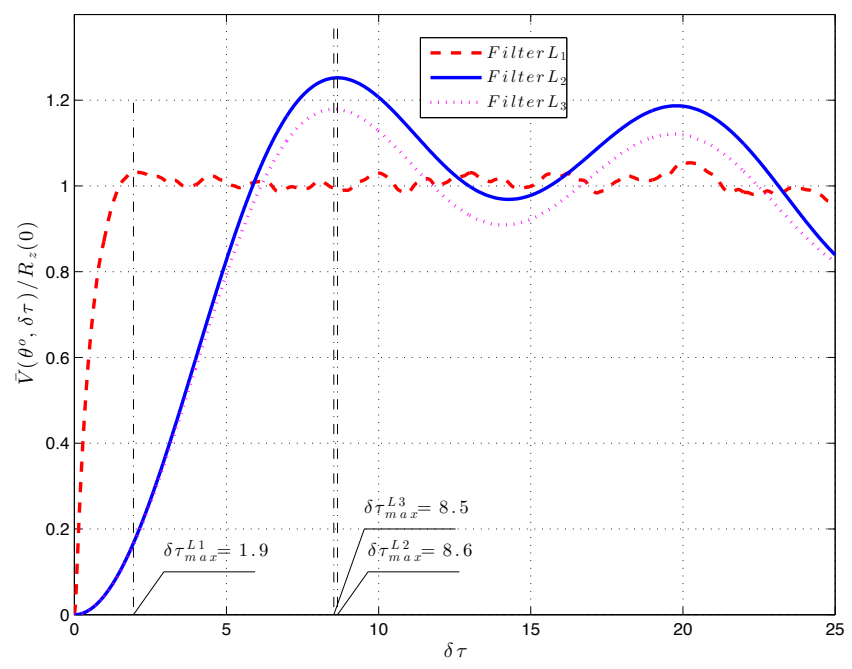

Fig. 1. Normalized cost function $\bar{V}\left(\boldsymbol{\theta}^{\circ}, \delta \tau\right) / R_{z}(0)$ for the 3 considered filters. 
The normalized cost function $\bar{V}\left(\boldsymbol{\theta}^{o}, \delta \tau\right) / R_{z}(0)$ versus $\delta \tau$ is plotted in Figure 1. It can be observed that without any low-pass filtering, $\delta \tau_{\max }^{\mathrm{L} 1} \approx 1.9$. When a low-pass filtering is applied, the admissible region is extended to $\delta \tau_{\text {max }}^{\mathrm{L} 2} \approx 8.6$ and $\delta \tau_{\max }^{\mathrm{L} 3} \approx 8.5$. The global convergence region is clearly enlarged by using a low-pass filter.

From Figure 1 , it can also be noticed that $\delta \tau_{\max }^{\mathrm{L} 2} \approx \delta \tau_{\max }^{\mathrm{L} 3} \approx$ $4.5 / \omega_{c}^{\mathrm{LF}}$. This means that $L_{2}(i \omega)$ and $L_{3}(i \omega)$ have almost the same effect. This phenomenon can be explained as follows: when $\omega_{c}^{\mathrm{LF}}$ is much smaller than the system bandwidth, if $\omega<\omega_{c}^{\mathrm{LF}}, G\left(i \omega, \boldsymbol{\theta}^{o}\right) \approx$ constant, its corresponding $T(i \omega)$ can also be regarded as an ideal low pass filter.

In the remainder of the paper, the ideal low-pass filter is used

$$
L(i \omega)= \begin{cases}1 & |\omega| \leq \omega_{c}^{\mathrm{LF}} \\ 0 & |\omega|>\omega_{c}^{\mathrm{LF}}\end{cases}
$$

Remark. 1: If the data are regularly sampled, the filtering operation can be carried out in the frequency domain by using the Fourier transform and its inverse, by adding a rectangular window to the frequency data. In the present case of irregularly sampled data, the non-uniform fast Fourier transform (NUFFT) and its inverse can be used (see e.g. [9]).

\section{Separable Method for Time-delay System IDENTIFICATION}

From the previous statements, $\boldsymbol{\theta}$ is estimated by the SRIVC method while $\tau$ is estimated by a numerical method. So it is natural to use a separable way to estimate $\boldsymbol{\theta}$ and $\tau$, when estimating each of them, the other is assumed to be fixed. The regression model once the additional filter $L(p)$ is applied, takes the following form

$$
\overline{\boldsymbol{y}}_{F}(\boldsymbol{\rho})=\overline{\boldsymbol{\Phi}}_{F}^{T}(\boldsymbol{\rho}, \tau) \boldsymbol{\theta}+\overline{\boldsymbol{\epsilon}}(\boldsymbol{\rho})
$$

where $\boldsymbol{\rho}=\left[\boldsymbol{\theta}^{T}, \tau\right]^{T}$, and $\bar{x}$ means $x$ is filtered by $L(p)$. The cost function to be minimized is given as

$$
\bar{J}_{N}(\boldsymbol{\rho})=\frac{1}{2(N-s+1)} \overline{\boldsymbol{\epsilon}}^{T}(\boldsymbol{\rho}) \overline{\boldsymbol{\epsilon}}(\boldsymbol{\rho})
$$

where $s$ is chosen to guarantee that $t_{s} \geq \tau$. The parameters can be estimated by the following separable method (see [7]). Compared with the original separable non-linear leastsquares method, we propose to estimate the linear part by the SRIVC method instead of the simple least-squares method, and perform a low-pass filtering operation in the first few iterations of the algorithm to widen the convergence region for the initial time-delay search.

Theorem 2: Let

$$
\hat{\boldsymbol{\theta}}(\tau)=\left[\overline{\boldsymbol{\Psi}}_{F}(\boldsymbol{\rho}) \overline{\boldsymbol{\Phi}}_{F}^{T}(\boldsymbol{\rho})\right]^{-1} \overline{\boldsymbol{\Psi}}_{F}(\boldsymbol{\rho}) \overline{\boldsymbol{y}}_{F}(\boldsymbol{\theta})
$$

Then $\hat{\tau}$ can be obtained as

$$
\begin{aligned}
& \underset{\rho}{\arg \min _{\boldsymbol{\rho}}} \bar{J}_{N}(\boldsymbol{\rho})=\arg \min _{\tau} \tilde{\bar{J}}_{N}(\tau) \\
& \hat{\tau}=\arg \min _{\tau} \tilde{\bar{J}}_{N}(\tau)
\end{aligned}
$$

where

$$
\tilde{\bar{J}}_{N}(\tau)=\left.\bar{J}_{N}(\boldsymbol{\rho})\right|_{\boldsymbol{\theta}=\hat{\boldsymbol{\theta}}(\tau)}
$$

The proposed Gauss-Newton method is then given as

$$
\begin{aligned}
\hat{\tau}^{j+1}= & \hat{\tau}^{j}-\mu^{j}\left[\nabla^{2} \tilde{\bar{J}}_{N}\left(\hat{\tau}^{j}\right)\right]^{-1} \nabla \tilde{\bar{J}}_{N}\left(\hat{\tau}^{j}\right) \\
\hat{\boldsymbol{\theta}}^{j+1}= & {\left[\overline{\boldsymbol{\Psi}}_{F}\left(\hat{\boldsymbol{\theta}}^{j}, \hat{\tau}^{j+1}\right) \overline{\boldsymbol{\Phi}}_{F}^{T}\left(\hat{\boldsymbol{\theta}}^{j}, \hat{\tau}^{j+1}\right)\right]^{-1} } \\
& \times \overline{\boldsymbol{\Psi}}_{F}\left(\hat{\boldsymbol{\theta}}^{j}, \hat{\tau}^{j+1}\right) \overline{\boldsymbol{y}}_{F}\left(\hat{\boldsymbol{\theta}}^{j}\right)
\end{aligned}
$$

where

$$
\begin{aligned}
& \nabla \tilde{\bar{J}}_{N}(\tau)=\frac{1}{N-s+1} \overline{\boldsymbol{\epsilon}}_{\tau}^{T} \overline{\boldsymbol{\epsilon}}(\boldsymbol{\rho}) \\
& \nabla^{2} \tilde{\bar{J}}_{N}(\tau) \approx \frac{1}{N-s+1}\left[\overline{\boldsymbol{\epsilon}}_{\tau}^{T} \overline{\boldsymbol{\epsilon}}_{\tau}-\overline{\boldsymbol{\epsilon}}_{\tau}^{T} \overline{\boldsymbol{\epsilon}}_{\boldsymbol{\theta}}\left(\overline{\boldsymbol{\epsilon}}_{\boldsymbol{\theta}}^{T} \overline{\boldsymbol{\epsilon}}_{\boldsymbol{\theta}}\right)^{-1} \overline{\boldsymbol{\epsilon}}_{\boldsymbol{\theta}}^{T} \overline{\boldsymbol{\epsilon}}_{\tau}\right] \\
& \boldsymbol{\epsilon}_{\tau}=\left.\frac{\partial \boldsymbol{\epsilon}(\boldsymbol{\rho})}{\partial \tau}\right|_{\boldsymbol{\rho}=\hat{\boldsymbol{\rho}}^{j}}=p G\left(p, \hat{\boldsymbol{\theta}}^{j}\right) \boldsymbol{u}\left(\hat{\tau}^{j}\right) \\
& \boldsymbol{\epsilon}_{\boldsymbol{\theta}}=\left.\frac{\partial \boldsymbol{\epsilon}(\boldsymbol{\rho})}{\partial \boldsymbol{\theta}}\right|_{\boldsymbol{\rho}=\hat{\boldsymbol{\rho}}^{j}}=-\boldsymbol{\Psi}_{F}^{T}\left(\hat{\boldsymbol{\rho}}^{j}\right)
\end{aligned}
$$

\section{A. The Proposed SRIVC-based Algorithm}

The complete algorithm (tdsrivc) for time-delay system identification can be summarized as

1) Initialization: Set boundaries $\Delta \tau_{\min }, \Delta \tau_{\max }, \tau_{\min }, \tau_{\max }$, the cut-off frequencies $\omega_{c}^{\mathrm{SVF}}, \omega_{c}^{\mathrm{LF}}$, and $\Delta \overline{\bar{J}}_{N}(\tau)_{\min }$. Initialize $F(p)$ by the following state-variable-filter (SVF)

$$
F(p)=\frac{1}{\left(p+\omega_{c}^{\mathrm{SVF}}\right)^{n}}
$$

Based on the initial value $\hat{\tau}^{1}$ and $F(p)$, use srivc (see e.g. [11] for more details about srivc method and the choice of $\omega_{c}^{\mathrm{SVF}}$ ) algorithm to compute $\hat{\boldsymbol{\theta}}^{1}$.

2) Initialization of the time-delay search for $\mathrm{j}=1: M$

a) Compute $\Delta \hat{\tau}^{j}$

$$
\Delta \hat{\tau}^{j}=-\left[\nabla^{2} \tilde{\bar{J}}_{N}\left(\hat{\tau}^{j}\right)\right]^{-1} \nabla \tilde{\bar{J}}_{N}\left(\hat{\tau}^{j}\right)
$$

b) Perform the following

i) Compute $\hat{\tau}^{j+1}=\hat{\tau}^{j}+\Delta \hat{\tau}^{j}$. If $\hat{\tau}^{j+1} \notin\left[\tau_{\min }, \tau_{\max }\right]$, let $\Delta \hat{\tau}^{j}=\Delta \hat{\tau}^{j} / 2$ and repeat this step.

ii) If $\left|\Delta \hat{\tau}^{j}\right|>\Delta \tau_{\max }$, let $\left|\Delta \hat{\tau}^{j}\right|=\Delta \tau_{\max }$.

If $\left|\Delta \hat{\tau}^{j}\right|<\Delta \tau_{\min }$, go to step (c).

iii) Estimate $\boldsymbol{\theta}$ using srive

Update auxiliary model and prefilter

$$
\begin{aligned}
& G\left(p, \hat{\boldsymbol{\theta}}^{j}\right)=\hat{B}\left(p, \hat{\boldsymbol{\theta}}^{j}\right) / \hat{A}\left(p, \hat{\boldsymbol{\theta}}^{j}\right) \\
& F\left(p, \hat{\boldsymbol{\theta}}^{j}\right)=1 / \hat{A}\left(p, \hat{\boldsymbol{\theta}}^{j}\right)
\end{aligned}
$$

Compute the filtered signals in

$$
\overline{\boldsymbol{\Psi}}_{F}\left(\hat{\boldsymbol{\theta}}^{j}, \hat{\tau}^{j+1}\right), \overline{\boldsymbol{\Phi}}_{F}\left(\hat{\boldsymbol{\theta}}^{j}, \hat{\tau}^{j+1}\right), \overline{\boldsymbol{y}}_{F}\left(\hat{\boldsymbol{\theta}}^{j}\right)
$$

Then estimate $\boldsymbol{\theta}$

$$
\begin{aligned}
\hat{\boldsymbol{\theta}}^{j+1}=[ & \left.\overline{\boldsymbol{\Psi}}_{F}\left(\hat{\boldsymbol{\theta}}^{j}, \hat{\tau}^{j+1}\right) \overline{\boldsymbol{\Phi}}_{F}^{T}\left(\hat{\boldsymbol{\theta}}^{j}, \hat{\tau}^{j+1}\right)\right]^{-1} \\
& \times \overline{\boldsymbol{\Psi}}_{F}\left(\hat{\boldsymbol{\theta}}^{j}, \hat{\tau}^{j+1}\right) \overline{\boldsymbol{y}}_{F}\left(\hat{\boldsymbol{\theta}}^{j}\right)
\end{aligned}
$$



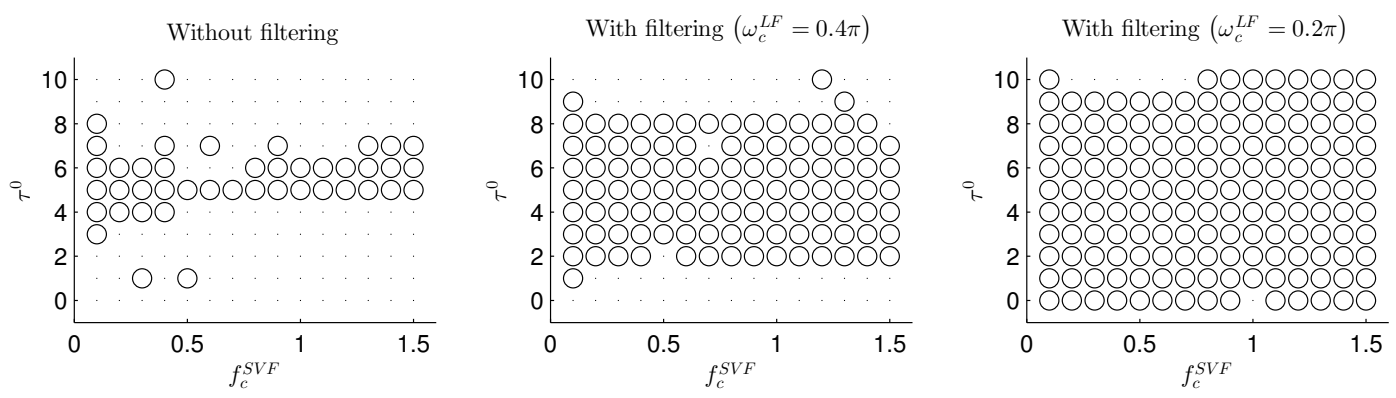

Fig. 2. Estimated results with and without low-pass filtering. Ring - fit $>99$. Dot - fit $\leq 99$.

iv) Compute $\tilde{\bar{J}}_{N}\left(\hat{\tau}^{j+1}\right)$.

If $\tilde{\bar{J}}_{N}\left(\hat{\tau}^{j+1}\right) \geq \tilde{\bar{J}}_{N}\left(\hat{\tau}^{j}\right)$, let $\Delta \hat{\tau}^{j}=\Delta \hat{\tau}^{j} / 2$ and go to step iii.

c) Check the stop condition, if $\tilde{\bar{J}}_{N}\left(\hat{\tau}^{j}\right)-$ $\tilde{\bar{J}}_{N}\left(\hat{\tau}^{j+1}\right) \geq \Delta \tilde{\bar{J}}_{N}(\tau)_{\min }$, go to step (a), else break.

end

3) Refined plant parameter and delay estimation

for $\mathrm{j}=M$ :convergence

Repeat step 2) without applying the low-pass filter $L(i \omega)$.

end

Note that in the above algorithm, tdsrivc runs in the end without low-pass filtering to obtain a refined estimate. The reason for using a two-step scheme is that the lowpass filtering increases the convergence region at the price of slightly decreasing the accuracy of the final estimate, so a refinement step is suggested. In addition, the maximum number of iterations $M$ for the initial time-delay search can be typically chosen between 5 to 10 .

\section{B. The Possibility of Handling Arbitrary Time-delay}

As discussed in Section III, when performing CT filtering, the actual input of the filter is a 'CT signal', which is reconstructed from the irregularly sampled data and the provided inter-sample behavior. With this reconstructed CT signal, it is possible to predict the output of a filter at any desired time-instant with the specified time-delay. In this way, the proposed method can therefore handle arbitrary time-delay identification.

\section{NUMERICAL EXAMPLES}

In this section, all the systems to be identified are assumed to be excited by a pseudo random binary sequence (PRBS), which is generated from a 9-stage shift register and the clock is set to $0.5 s$. The time-varying sampling period $h_{k}$ is assumed to be uniformly distributed in the following interval

$$
h_{k} \sim U[0.01,0.09] s
$$

The stopping rule for the iterative algorithm is that the relative change in $\tilde{\bar{J}}_{N}(\hat{\tau})$ or $\tilde{J}_{N}(\hat{\tau})$ is lower than $10^{-4}$; the maximum iteration number of stage 2 ) is $M=10$; the timedelay boundaries are $\tau_{\min }=0$ and $\tau_{\max }=10$.

\section{A. Noise-free Case}

To illustrate the positive effect of the low-pass filtering strategy on the convergence performance, let us first consider the noise-free situation. The second-order system to be studied is given as

$$
y(t)=\frac{2}{0.25 p^{2}+0.7 p+1} u(t-5)
$$

Different initializations for the cut-off frequency of the initial filter $\omega_{c}^{\mathrm{SVF}}$ used in the SRIVC method and $\tau^{0}$ are tested one by one

$$
\begin{aligned}
\omega_{c}^{\mathrm{SVF}} & =\{0.2 \pi, 0.4 \pi, 0.6 \pi, \cdots, 3 \pi\} \mathrm{rad} / \mathrm{s} \\
\tau^{0} & =\{0,1,2, \cdots, 10\} \mathrm{s}
\end{aligned}
$$

for two cut-off frequency of the low-pass filter $\left(\omega_{c}^{\mathrm{LF}}=\right.$ $\left.0.4 \pi, \omega_{c}^{\mathrm{LF}}=0.2 \pi\right)$. For each run, the number of observations is $N=10,000$. The criterion of global convergence is fit $>99$, where fit is the normalized root-mean-square error defined as follows

$$
\text { fit }=100\left(1-\frac{\|y(t)-\hat{x}(t)\|_{2}}{\|y(t)-\mathbb{E}\{y(t)\}\|_{2}}\right)
$$

where $\hat{x}\left(t_{k}\right)$ is the noise-free response of the estimated model, $y\left(t_{k}\right)$ is the sampled output.

Two identification schemes has been tested:

A. Identification without low-pass filtering.

B. Identification with low-pass filtering.

The estimated results are presented in Fig. 2 for the two chosen values of $\omega_{c}^{\mathrm{LF}}$. From this figure we see that the results for scheme A (no filtering) are very poor. Convergence is reached when $\tau^{0}$ lies in the interval $[4,6]$. Meanwhile, a SVF filter with lower cut-off frequency slightly increases the probability of global convergence. The results for Scheme B are overall quite satisfying. It can be noted that when $\omega_{c}^{\mathrm{LF}}$ decreases, the global minimum is more easy to access.

One question remains here to know how $\omega_{L}$ should be chosen. It has concluded that the global minimum is easier to access for smaller values of $\omega_{c}^{\mathrm{LF}}$. Then, one may ask, can $\omega_{c}^{\mathrm{LF}}$ be arbitrarily small? The answer is no, the reason can be given as: smaller values for $\omega_{c}^{\mathrm{LF}}$ mean that more information of the sampled data is removed, which can deteriorate the plant parameter estimation. A rule of thumb can be to choose the cut-off frequency of the ideal low-pass filter as $\frac{1}{10}$ to $\frac{1}{2}$ of the system bandwidth. 


\begin{tabular}{|c|c|c|c|c|c|c|c|c|}
\hline True system & Low-pass filtering & $\hat{a}_{0}$ & $\hat{a}_{1}$ & $\hat{b}_{0}$ & $\hat{b}_{1}$ & $\hat{\tau}$ & $N_{\text {iter }}$ & $P$ \\
\hline \multirow{2}{*}{ 1: $\frac{2 e^{-5 s}}{2 s+1}$} & No & $\begin{array}{c}2.0003 \\
\pm 0.0153\end{array}$ & - & $\begin{array}{c}2.0003 \\
\pm 0.0114\end{array}$ & & $\begin{array}{c}4.9999 \\
\pm 0.0030\end{array}$ & 10.2 & $45 \%$ \\
\hline & Yes & $\begin{array}{c}1.9994 \\
\pm 0.0160\end{array}$ & $\longrightarrow$ & $\begin{array}{c}1.9992 \\
\pm 0.0114\end{array}$ & & $\begin{array}{c}5.0001 \\
\pm 0.0033\end{array}$ & 10.4 & $96 \%$ \\
\hline \multirow{2}{*}{ 2: $\frac{3 e^{-5 s}}{0.25 s^{2}+s+1}$} & No & $\begin{array}{c}0.2496 \\
\pm 0.0056\end{array}$ & $\begin{array}{c}0.9991 \\
\pm 0.0068\end{array}$ & $\begin{array}{c}2.9995 \\
\pm 0.0137\end{array}$ & & $\begin{array}{c}5.0003 \\
\pm 0.0048\end{array}$ & 6.2 & $42 \%$ \\
\hline & Yes & $\begin{array}{c}0.2496 \\
\pm 0.0069\end{array}$ & $\begin{array}{c}0.9983 \\
\pm 0.0072\end{array}$ & $\begin{array}{c}2.9972 \\
\pm 0.0152\end{array}$ & & $\begin{array}{c}5.0005 \\
\pm 0.0058\end{array}$ & 10.1 & $100 \%$ \\
\hline \multirow{2}{*}{$3: \frac{2 e^{-5 s}}{0.25 s^{2}+0.7 s+1}$} & No & $\begin{array}{c}0.2499 \\
\pm 0.0041\end{array}$ & $\begin{array}{c}0.6987 \\
\pm 0.0043\end{array}$ & $\begin{array}{c}1.9983 \\
\pm 0.0087\end{array}$ & & $\begin{array}{c}4.9997 \\
\pm 0.0050\end{array}$ & 6.5 & $57 \%$ \\
\hline & Yes & $\begin{array}{c}0.2498 \\
\pm 0.0039\end{array}$ & $\begin{array}{c}0.6988 \\
\pm 0.0049\end{array}$ & $\begin{array}{c}1.9984 \\
\pm 0.0099\end{array}$ & & $\begin{array}{c}5.0002 \\
\pm 0.0045\end{array}$ & 9.9 & $100 \%$ \\
\hline \multirow{2}{*}{$4: \frac{(-4 s+1) e^{-5 s}}{9 s^{2}+2.4 s+1}$} & No & $\begin{array}{c}9.0020 \\
\pm 0.0581\end{array}$ & $\begin{array}{c}2.3991 \\
\pm 0.0211\end{array}$ & $\begin{array}{l}-4.0029 \\
\pm 0.0271\end{array}$ & $\begin{array}{c}0.9995 \\
\pm 0.0143\end{array}$ & $\begin{array}{c}4.9993 \\
\pm 0.0056\end{array}$ & 8.5 & $42 \%$ \\
\hline & Yes & $\begin{array}{c}8.9923 \\
\pm 0.0482\end{array}$ & $\begin{array}{c}2.3964 \\
\pm 0.0193\end{array}$ & $\begin{array}{l}-3.9972 \\
\pm 0.0286\end{array}$ & $\begin{array}{c}0.9973 \\
\pm 0.0127\end{array}$ & $\begin{array}{c}5.0000 \\
\pm 0.0056\end{array}$ & 11.5 & $84 \%$ \\
\hline
\end{tabular}

TABLE I

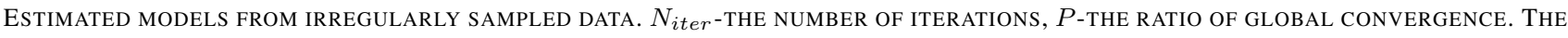
MEAN VALUE, THE STANDARD DEVIATION AND $N_{i t e r}$ ARE COMPUTED FROM THE SUCCESSFUL ESTIMATED MODELS.

\section{B. Noisy-output Case}

In this subsection, it is assumed that the measured output is corrupted by a discrete-time white noise, the signal to noise ratio $(\mathrm{SNR})$ is $15 \mathrm{~dB}$. The $\mathrm{SNR}$ is defined as

$$
\mathrm{SNR}=10 \log \frac{P_{x}}{P_{e}}
$$

where $P_{x}$ and $P_{e}$ represent the average power of the noisefree signal $x\left(t_{k}\right)$ and the disturbance $e\left(t_{k}\right)$. The number of observed data is $N=2000$. The cut-off frequency of the initial SVF filter is fixed to $\omega_{c}^{\mathrm{SVF}}=2 \pi$.

Due to the fact that the time-delay of the system to be studied is lower than 10 , any choice of $\omega_{c}^{\mathrm{LF}}$ leads to $\delta \tau_{\max } \geq 5$ is reasonable, here we choose $\omega_{c}^{\mathrm{LF}}=0.1 \pi$. The initial guess of the time-delay is assumed to be uniformly distributed in the following interval

$$
\tau^{0} \sim U[0,10] s
$$

In this noisy-output case, the criterion for global convergence fit satisfies the following inequality

$$
\begin{aligned}
\text { fit } & \leq 100\left(1-\frac{\sqrt{P_{e}}}{\sqrt{P_{x}+P_{e}}}\right) \\
& =100\left[1-\left(1+10^{\mathrm{SNR} / 10}\right)^{-1 / 2}\right] \approx 82.5
\end{aligned}
$$

The criterion for 'success' is fit $>81$. The estimated results are given in Table I. This table shows that when no filter is applied (scheme A), the global convergence ratio is less than $60 \%$, while the success ratio surges to $84 \% \sim 100 \%$ when a filtering option (scheme B) is adopted. The good performance does not come for free, the cost is the slight increase of the iteration number to converge.

\section{CONCLUSION}

In this paper, identification of continuous-time system with arbitrary time-delay from irregularly sampled data has been considered. The proposed algorithm estimate the plant parameters and the time-delay in a separable way, more precisely, the plant is estimated by the SRIVC method and the time-delay is estimated by the Gauss-Newton method, respectively. An ideal low-pass filter is introduced to extend the global convergence region. Numerical examples have been used to show that, under mild initializations, the global convergence ratio can be increased to $80 \% \sim 100 \%$ after the use of a low-pass filter.

\section{REFERENCES}

[1] S. Ahmed, B. Huang, and S. L. Shah. Parameter and delay estimation of continuous-time models using a linear filter. Journal of Process Control, 16:323-331, 2006.

[2] F. Chen, H. Garnier, M. Gilson, J. C. Agüero, and B. I. Godoy. Identification of continuous-time transfer function models from nonuniformly sampled data in presence of colored noise. In The 19th IFAC World Congress, Cape Town, South Africa, 24-29 August 2014

[3] G. Ferretti, C. Maffezzoni, and R. Scattolini. On the identifiability of the time delay with least-squares methods. Automatica, 32(3):449453, 1996.

[4] H. Garnier, M. Gilson, P. C. Young, and E. Huselstein. An optimal IV technique for identifying continuous-time transfer function mode of multiple input systems. Control Engineering Practice, 46(15):471486, 2007.

[5] H. Garnier and L. Wang(Eds.). Identification of Continuous-time Models from Sampled Data. Springer-Verlag, London, 2008.

[6] L. Ljung. System Identification - Theory for the User. Prentice-Hall, Upper Saddle River, 2nd edition edition, 1999.

[7] L. S. H. Ngia. Separable nonlinear least-squares methods for efficient off-line and on-line modeling of systems using Kautz and Laguerre filters. IEEE Transactions on Circuits and Systems II: Analog and Digital Signal Processing, 48(6):562-579, 2001

[8] B. Ni, D. Xiao, and S. L. Shah. Time delay estimation for MIMO dynamical systems - With time - frequency domain analysis. Journal of Process Control, 1(20):83-94, 2010.

[9] A. F. Ware. Fast approximate Fourier transforms for irregularly spaced data. SIAM Review, 40(4):838-856, 1998.

[10] Z. Yang, H. Iemura, S. Kanae, and K. Wada. Identification of continuous-time systems with multiple unknown time delays by global nonlinear least-squares and instrumental variable methods. Automatica, 43(7):1257-1264, 2007.

[11] P. C. Young, H. Garnier, and M. Gilson. Refined instrumental variable identification of continuous-time hybrid Box-Jenkins models. In Identification of Continuous-time Models from Sampled Data (H. Garnier and L. Wang (Eds.)), pages 91-132, London, 2008. SpringerVerlag.

[12] P. C. Young and A. J. Jakeman. Refined instrumental variable methods of time-series analysis: Part III, extensions. International Journal of Control, 31:741-764, 1980. 